\title{
A current noise reduction technique in chopper instrumentation amplifier for high-impedance sensors
}

\author{
Ippei Akita $^{1 \text { a) }}$ and Makoto Ishida ${ }^{1,2}$ \\ ${ }^{1}$ Department of Electrical and Electronic Information Engineering, \\ Toyohashi University of Technology, \\ 1-1 Hibarigaoka, Tempaku-cho, Toyohashi, Aichi 441-8580, Japan \\ ${ }^{2}$ Electronics-Inspired Interdisciplinary Research Institute (EIIRIS), \\ Toyohashi University of Technology, \\ 1-1 Hibarigaoka, Tempaku-cho, Toyohashi, Aichi 441-8580, Japan \\ a)ippei.akita@ieee.org
}

Abstract: A current noise reduction technique in chopper instrumentation amplifier (CIA) for high-impedance sensor is presented. The proposed technique is based on a time gating method to reduce time varying shot noise which is induced by channel charge injection of chopper switch transistors. An implemented CIA with the proposed time gating technique achieves more than $80-\%$ noise reduction capability.

Keywords: chopper stabilization, instrumentation amplifiers, input current noise

Classification: Integrated circuits

\section{References}

[1] C. C. Enz and G. C. Temes: Proc. IEEE 84 (1996) 1584. DOI:10.1109/5.542410

[2] I. Akita and M. Ishida: ISSCC Dig. Tech. Papers (2013) 178. DOI:10.1109/ ISSCC.2013.6487689

[3] I. Akita and M. Ishida: Analog Integr. Circuits Signal Process. 81 (2014) 571. DOI:10.1007/s10470-014-0371-4

[4] J. Xu, Q. Fan, J. H. Huijsing, C. Van Hoof, R. F. Yazicioglu and K. A. Makinwa: IEEE J. Solid-State Circuits 48 (2013) 1575. DOI:10.1109/JSSC.2013.2253217

[5] D. Drung and J. H. Storm: IEEE Trans. Instrum. Meas. 60 (2011) 2347. DOI:10. 1109/TIM.2011.2114030

\section{Introduction}

Chopper instrumentation amplifiers (CIAs) provide a good low-frequency noise characteristic as sensor front-end circuits $[1,2,3]$. The input-referred current noise performance is degraded when the output impedance of sensors is high because the channel charge of input chopper switch transistors generates shot noise as shown in 
Fig. 1 [4]. Assuming use of a CIA topology in Fig. 1a, such channel charge flows into a sensor with impedance $R_{i n}$, resulting in a large current spike $I_{q}(t)$ synchronizing chopper clock $\phi_{c h}$ as shown in Fig. 1b. Therefore, $I_{q}(t)$ causes shot noise which has been observed in measurement and characterized as input-referred current noise; $\sqrt{2 q I_{q} \Delta f}$ where $q$ and $\Delta f$ are elementary charge and equivalent bandwidth, respectively $[4,5]$. Note that shot noise follows the Poisson distribution involved with $I_{q}(t)$ and it proportional to chopper frequency in CIAs. In [4], for simplifying its analysis, however, $I_{q}(t)$ is considered as its average value, $\overline{I_{q}(t)}$, as shown in Fig. 1b. Although this approach enables a simple circuit simulation and it provides well-matched results with measurement ones, shot noise should be modeled as time-varying noise exactly in this case because $I_{q}(t)$ is dynamic current, not static one $\overline{I_{q}(t)}$ as shown in Fig. 1c.

Focusing on this fact, this letter presents a simple current noise reduction technique by using time gating technique which nulls output during $I_{q}(t)$ arises, resulting in a low-noise CIAs for high-impedance sensor devices.

(a)

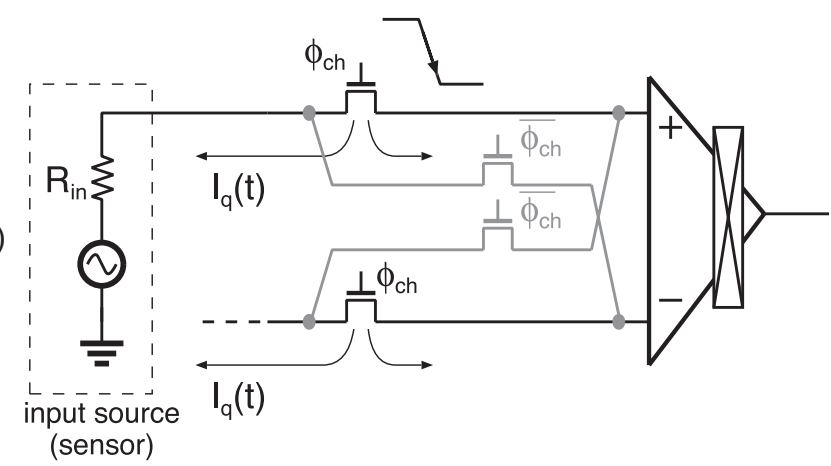

(b)
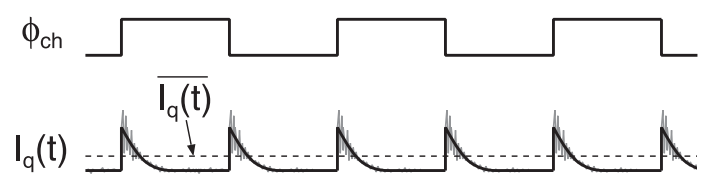

(c) shot noise

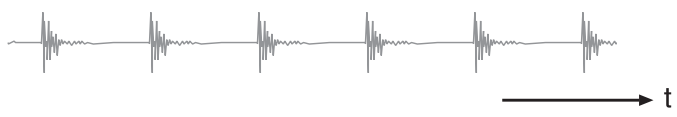

Fig. 1. Current noise in chopper instrumentation amplifier (CIA): (a) Impulse current $I_{q}(t)$ due to channel charge injection, (b) Its waveforms, and (c) Time-variant shot noise waveform due to $I_{g}(t)$.

\section{CIA with time gating technique}

Fig. 2 shows the proposed noise-gating CIA and its timing diagram of the clock signals. The CIA consists of three opamps and chopper switches driven by a clock $\phi_{c h}$ the frequency of which is $f_{c h}$. In this design, the chopper is adopted to the first stage of the CIA. As mentioned above, the current spike $I_{q}(t)$ flows at the instance of the transition of $\phi_{c h}$, and then shot noise modulated by $I_{q}(t)$ arises. In order to cancel the influence of the modulated noise, a switch is introduced between the 
differential terminals of the first stage output as shown in Fig. 2. This switch is driven by a clock $\phi_{\text {gate }}$ which has a half period of $\phi_{c h}$, and the positive edge of which is synchronizing with the transition of $\phi_{c h}$. Therefore, since the output voltage of the first stage goes into common-mode one during $\phi_{\text {gate }}$ goes high, the output of the second stage, $V_{o, a m p}$, becomes zero and thus the time-varying shot noise can be almost cancelled.

In this time gating technique, although the degradation of the signal integrity due to shot noise is avoided, a desired effective signal is simultaneously decreases. This means that the voltage gain of the CIA is inversely proportional to the duty ratio of $\phi_{\text {gate }}$, which is derived as $2 \Delta T_{\text {gate }} / T_{c h}$ where $\Delta T_{\text {gate }}$ is the pulse width of $\phi_{g a t e}$ and $T_{c h}=1 / f_{c h}$ is a period of $\phi_{c h}$. Therefore, the noise performance of the CIA should be evaluated by the input-referred current noise. Since the shot noise is time-variant and it attenuates in an exponential function manner, an optimum duty ratio exist for time gating. If the ratio goes small, the time gating becomes less effective. On the other hand, the input-referred current noise increases for a large duty ratio because the effective voltage gain of the CIA decreases. Note that a low pass filter (LPF) is required to suppress modulated images due to time gating.

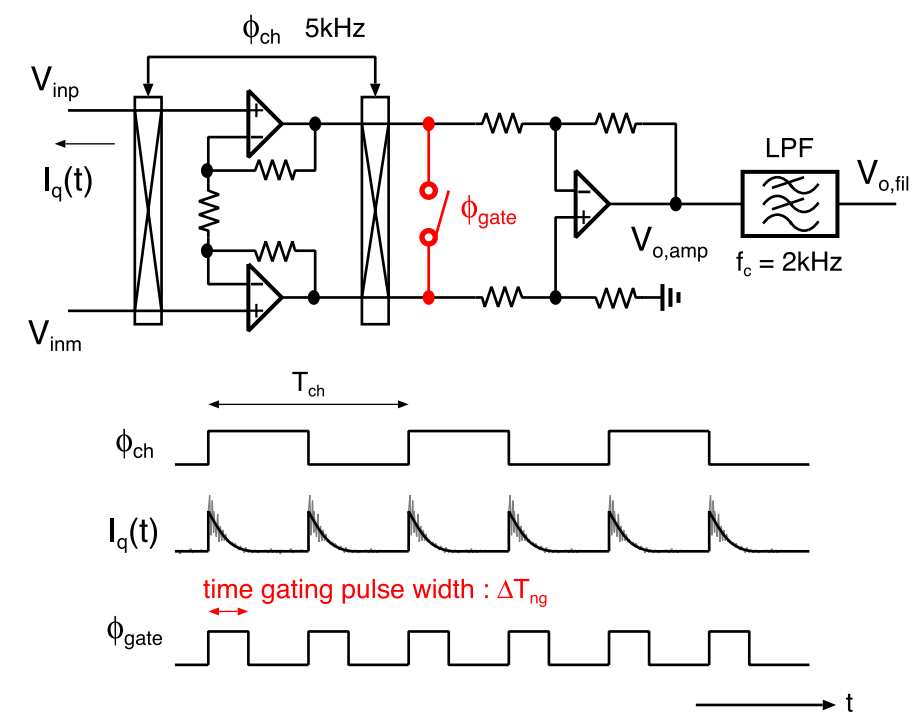

Fig. 2. Proposed noise-gating CIA and clock timing diagram

\section{Measurement results}

In order to confirm the effectiveness of the proposed time gating technique, a CIA shown in Fig. 2 is designed and measured. The CIA is composed of some discrete devices, such as opamps OPA2132, switches AD7512DI, some resistors and capacitors. The supply voltages are $\pm 15 \mathrm{~V}$ and the common-mode one is the ground. The frequencies of the chopper clock $\phi_{c h}$ and the time gating clock $\phi_{\text {gate }}$ are $5 \mathrm{kHz}$ and $10 \mathrm{kHz}$, respectively. SR640 is used as the following LPF and its cut-off frequency is $2 \mathrm{kHz}$. The voltage gain, $A_{v}=V_{o, a m p} /\left(V_{\text {inp }}-V_{\text {inm }}\right)$, is set to $44 \mathrm{~dB}$ when the time gating is in disable. The current noise of the CIA is measured by using resistors, $R_{\text {in }}$ 's, which are connected to two input terminals, and observing the 
output voltage noise, where $R_{\text {in }}=1.2 \mathrm{M} \Omega$ and the contribution of its thermal noise is subtracted [4].

Fig. 3 shows the waveforms for a $900-\mathrm{Hz} 25-\mathrm{mV}$ sinusoidal input voltage where the duty ration of $\phi_{\text {gate }}$ is $50 \%$. As seen from the CIA output waveform, $V_{o, a m p}$, its voltage is periodically reset to the common-mode with synchronizing $\phi_{\text {gate }}$. Finally, the modulated images in $V_{o, a m p}$ is filtered out by the LPF, resulting in $V_{o, f i l}$.

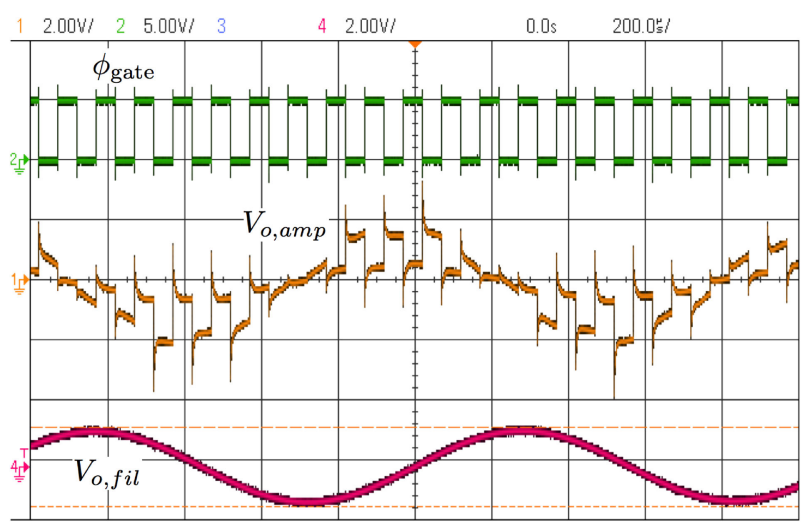

Fig. 3. Waveforms for $25-\mathrm{mV} 900-\mathrm{Hz}$ sinusoidal input

Fig. 4a shows the power spectrum density (PSD) for the input-referred current noise, in which the noise floor lowers as the duty ratio increases. Therefore, it is confirmed that the capability of current noise suppression depends on the duty ratio of $\phi_{\text {gate }}$. Fig. $4 \mathrm{~b}$ shows relation between the duty ratio and the input-referred r.m.s current noise which is integrated from 10 to $400 \mathrm{~Hz}$ without the power line harmonics. As seen from results, the duty ratio of more than $25 \%$ provides a low input-referred current noise. On the other hand, a less effectiveness is confirmed for higher duty ratio, more than $80 \%$, because the noise in the later of $\phi_{\text {gate }}$ period is smaller than the former, and the effect of decreasing voltage gain is larger than that of decreasing noise, resulting in degradation of current noise characteristic. Therefore, in this designed CIA the optimum duty ration of $\phi_{\text {gate }}$ is about from 25 to $80 \%$, and in this situation, the CIA with the proposed technique achieves more than $80-\%$ reduction capability. Furthermore, if a bootstrapped switch is also used for input chopper one, more reduction could be realized because a limited gate overdrive voltage of chopper switch reduces channel charge injection [4].

\section{Conclusion}

A time gating technique in CIA for high-impedance input sources is proposed, which is a simple method to reduce the time-varying shot noise induced by channel charge injection of chopper switches. For the proof of concept, a CIA using discrete components is implemented and the effectiveness of decreasing input-referred current noise is confirmed, achieving in more than $80-\%$ noise reduction capability. 


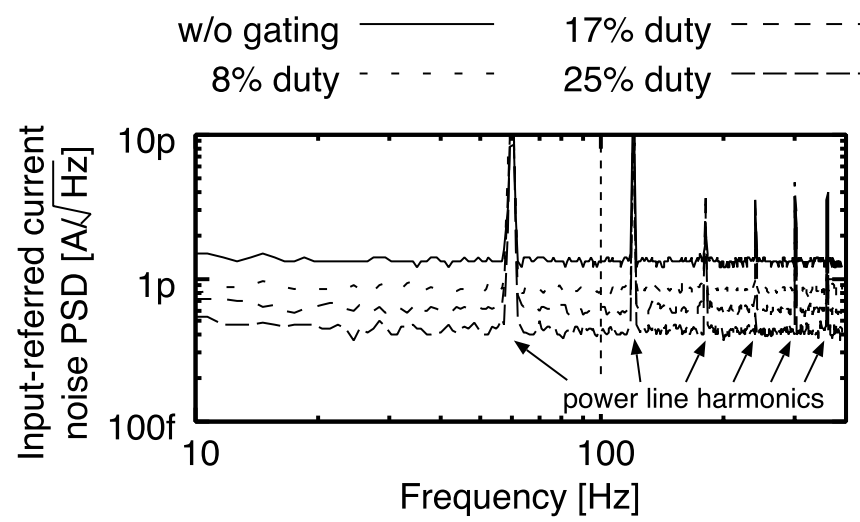

(a)

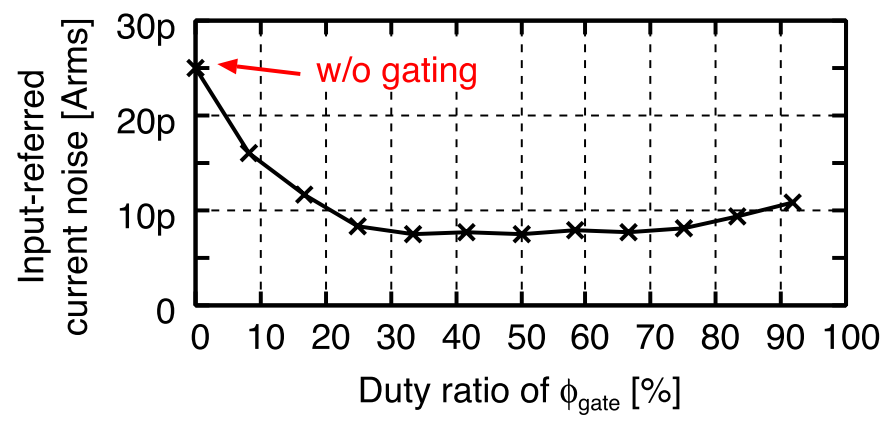

(b)

Fig. 4. Measured input-referred current noise for several duty ratio of $\phi_{\text {gate }}:$ (a) Power spectral density and (b) Integrated current noise from 10 to $400 \mathrm{~Hz}$.

\section{Acknowledgments}

This work has been supported in part by MEXT Grant-in-Aid for Scientific Research (25820141 and 15H05525), Ozawa \& Yoshikawa Memorial Electronics Research Foundation, and Tateisi Science and Technology Foundation. 